\title{
Convex and Exact Games with Non-transferable Utility
}

Citation for published version (APA):

Csóka, P., Herings, P. J. J., Kóczy, L. Á., \& Pintér, M. (2009). Convex and Exact Games with Nontransferable Utility. Maastricht University School of Business and Economics. METEOR Research Memorandum No. 031 https://doi.org/10.26481/umamet/2009031

Document status and date:

Published: 01/01/2009

DOI:

10.26481/umamet/2009031

Document Version:

Publisher's PDF, also known as Version of record

\section{Please check the document version of this publication:}

- A submitted manuscript is the version of the article upon submission and before peer-review. There can be important differences between the submitted version and the official published version of record.

People interested in the research are advised to contact the author for the final version of the publication, or visit the DOI to the publisher's website.

- The final author version and the galley proof are versions of the publication after peer review.

- The final published version features the final layout of the paper including the volume, issue and page numbers.

Link to publication

\footnotetext{
General rights rights.

- You may freely distribute the URL identifying the publication in the public portal. please follow below link for the End User Agreement:

www.umlib.nl/taverne-license

Take down policy

If you believe that this document breaches copyright please contact us at:

repository@maastrichtuniversity.nl

providing details and we will investigate your claim.
}

Copyright and moral rights for the publications made accessible in the public portal are retained by the authors and/or other copyright owners and it is a condition of accessing publications that users recognise and abide by the legal requirements associated with these

- Users may download and print one copy of any publication from the public portal for the purpose of private study or research.

- You may not further distribute the material or use it for any profit-making activity or commercial gain

If the publication is distributed under the terms of Article $25 \mathrm{fa}$ of the Dutch Copyright Act, indicated by the "Taverne" license above, 


\section{Maastricht University}

Péter Csóka, P. J ean-J acques Herings, László A. Kóczy, Miklós Pintér

Convex and Exact Games with Non-transferable Utility

$\mathrm{RM} / 09 / 031$

\section{METEOR}

Faculty of Economics and Business Administration Maastricht Research School of Economics

of Technology and Organization

P.O. Box 616

NL - 6200 MD Maastricht

The Netherlands 


\title{
Convex and Exact Games with Non-transferable Utility
}

\author{
Péter Csóka* P. Jean-Jacques Herings ${ }^{\dagger} \quad$ László Á. Kóczy \\ Miklós Pintér ${ }^{\S}$
}

June 20, 2009

\begin{abstract}
We generalize exactness to games with non-transferable utility (NTU). In an exact game for each coalition there is a core allocation on the boundary of its payoff set.

Convex games with transferable utility are well-known to be exact. We study five generalizations of convexity in the NTU setting. We show that each of ordinal, coalition merge, individual merge and marginal convexity can be unified under NTU exactness. We provide an example of a cardinally convex game which is not NTU exact.

Finally, we relate the classes of $\Pi$-balanced, totally $\Pi$-balanced, NTU exact, totally NTU exact, ordinally convex, cardinally convex, coalition merge convex, individual merge convex and marginal convex
\end{abstract}

*Department of Finance, Corvinus University of Budapest. The author would like to thank Corvinus University of Budapest for the "Kutatási kiválósági" research scholarship. peter.csoka@uni-corvinus.hu

${ }^{\dagger}$ Corresponding author. Department of Economics, Maastricht University, P.O. Box 616, 6200 MD, Maastricht, The Netherlands. The author would like to thank the Netherlands Organisation for Scientific Research (NWO) for financial support. p.herings@algec.unimaas.nl

${ }^{\ddagger}$ Keleti Faculty of Economics, Budapest Tech and Department of Economics, Maastricht University. The author thanks the funding of the OTKA (Hungarian Fund for Scientific Research) under the project NF-72610 and of the European Commission under a Marie Curie Reintegration Grant (PERG-GA-2008-230879). koczy. laszlo@kgk. bmf .hu

$\S$ Department of Mathematics, Corvinus University of Budapest. The author thanks the OTKA (Hungarian Fund for Scientific Research) and the János Bolyai Research Scholarship of the Hungarian Academy of Sciences for financial support. miklos.pinter@uni-corvinus.hu 
games to one another.

Keywords: NTU Games, Exact Games, Convex Games

JEL Classification: C71

\section{Introduction}

Convex cooperative games with transferable utility (TU) introduced by Shapley (1971) arise from a wide range of applications. Airport games (Littlechild and Owen, 1973), bankruptcy games (Aumann and Maschler, 1985), sequencing games (Curiel, Pederzoli, and Tijs, 1989) and standard tree games (Granot, Maschler, Owen, and Zhu, 1996) are all convex.

Convex TU games are exact (Schmeidler, 1972). In an exact game for each coalition there is a core allocation such that the coalition only gets its stand-alone value. Calleja, Borm, and Hendrickx (2005) show that the class of multi-issue allocation games coincides with the class of non-negative exact games. Csóka, Herings, and Kóczy (2008) demonstrate that the class of exact games equals the class of risk allocation games with no aggregate uncertainty.

Although transferable utility has proved itself to be a very valuable workhorse, it is a restrictive assumption, and generalizations of convexity and exactness to the non-transferable utility case are highly desired. Vilkov (1977) and Sharkey (1981) have extended convexity to games with non-transferable utility (NTU) to define ordinal and cardinal convexity, respectively. Hendrickx, Borm, and Timmer (2002) analyze coalition merge convexity, individual merge convexity, and marginal convexity in an NTU setting. The aforementioned five classes of NTU convex games do not coincide in general. The only general result (restated in this paper as Theorem 2.14) is that coalition merge convexity implies individual merge convexity, and individual merge convexity implies marginal convexity.

In this paper we generalize exactness to the NTU setting. In an exact NTU game for each coalition there is a core element on the boundary of its payoff set, meaning that this coalition does not necessarily benefit from the gains of forming the grand coalition in an allocation which is robust against all coalitional deviations. We show that each of ordinal, coalition merge, individual merge, and marginal convexity implies NTU exactness. We provide an example of a cardinally convex game which is not NTU exact.

The structure of the paper is as follows. We start with the notation and the necessary definitions for TU and NTU games. In Section 3 we define NTU exactness and from this perspective analyze the five classes of NTU 
convex games. In Section 4 we conclude by relating the various classes of NTU games to one another.

\section{Notation, Definitions, Existing Results}

Let $N=\{1, \ldots, n\}$ denote the finite set of players, $2^{N}=\{C \mid C \subseteq N\}$ is the power set of $N, \mathcal{N}=2^{N} \backslash\{\emptyset\}$ is the collection of coalitions, the nonempty subsets of $N$. Let $\mathbb{R}$ denote the set of all real numbers. $\mathbb{R}^{N}$ is the $n$-dimensional Euclidean space generated by the set of players. An element of $\mathbb{R}^{N}$ is denoted by a vector $x=\left(x_{i}\right)_{i \in N}$. For a coalition $C \in \mathcal{N}$, let $x^{C}=\left(x_{i}\right)_{i \in C}$ denote the restriction of $x$ on $C$. For $x, y \in \mathbb{R}^{N}, y \geq x$ denotes $y_{i} \geq x_{i}$ for all $i \in N$, and $y \gg x$ denotes $y_{i}>x_{i}$ for all $i \in N$.

Let $\Delta^{N}$ denote the unit simplex in $\mathbb{R}^{N}, \Delta^{N}=\left\{x \in \mathbb{R}^{N} \mid x_{i} \geq 0, \sum_{i \in N} x_{i}=\right.$ $1\}$. For every $C \in \mathcal{N}$ define the set $\Delta^{C}=\left\{x \in \Delta^{N} \mid \sum_{i \in C} x_{i}=1\right\}$, and let $\Delta$ be a Cartesian product of $\Delta^{C}$ over all $C \in \mathcal{N}$. Let $\Delta^{\mathcal{N}}$ denote the unit simplex in $\mathbb{R}^{\mathcal{N}}, \Delta^{\mathcal{N}}=\left\{\lambda \in \mathbb{R}^{\mathcal{N}} \mid \lambda^{C} \geq 0, \sum_{C \in \mathcal{N}} \lambda^{C}=1\right\}$.

For a set $A \subseteq \mathbb{R}^{N}$, the symbols cl $A, \partial A$ and int $A$ denote, respectively, the closure, the boundary and the interior of $A$. For $x \in \mathbb{R}^{N}, x \in \operatorname{cl} A$ if there exists a sequence $\left(x^{k}\right)_{k \in \mathbb{N}}$ with $x^{k} \in A$ for all $k \in \mathbb{N}$ and $\left(x^{k}\right)_{k \in \mathbb{N}} \rightarrow x$; $x \in \partial A$ if $x \in \operatorname{cl} A \cap \operatorname{cl}\left(\mathbb{R}^{N} \backslash A\right)$; and $x \in \operatorname{int} A$ if $x \in A \backslash \partial A$.

\subsection{Transferable Utility Games}

$A$ value function $v: 2^{N} \rightarrow \mathbb{R}$ satisfying $v(\emptyset)=0$ gives rise to a cooperative game with transferable utility (TU game, for short) $(N, v)$. Let $\Gamma^{\mathrm{TU}}$ denote the set of TU games with $n$ players. A utility allocation is a vector $x \in$ $\mathbb{R}^{N}$, where $x_{i}$ is the payoff of player $i \in N$. For a coalition $C \in \mathcal{N}$, let $x(C)=\sum_{i \in C} x_{i}$. An allocation $x \in \mathbb{R}^{N}$ is called efficient if $x(N)=v(N)$, individually rational if $x_{i} \geq v(\{i\})$ for all $i \in N$, and coalitionally rational if $x(C) \geq v(C)$ for all $C \in \mathcal{N}$. The core is the set of efficient and coalitionally rational allocations.

Shapley (1971) and Schmeidler (1972) introduce exact TU games.

Definition 2.1. A TU game $(N, v)$ is exact if for each $C \in 2^{N}$ there exists a core allocation $x$ such that $x(C)=v(C)$.

Let $\Gamma_{\mathrm{e}}^{\mathrm{TU}}$ denote the class of exact TU games with the player set $N$. Convex TU games (Shapley, 1971) can be defined and characterized as follows. 
Definition 2.2. A TU game $(N, v)$ is convex if it satisfies the following three equivalent conditions:

$$
\begin{aligned}
\forall S, T \in 2^{N}: v(S)+v(T) & \leq v(S \cup T)+v(S \cap T), \\
\forall U \in 2^{N} ; \forall S \subsetneq T \subseteq N \backslash U: v(S \cup U)-v(S) & \leq v(T \cup U)-v(T), \\
\forall i \in N ; \forall S \subsetneq T \subseteq N \backslash\{i\}: v(S \cup\{i\})-v(S) & \leq v(T \cup\{i\})-v(T) .
\end{aligned}
$$

Let $\Gamma_{\mathrm{c}}^{\mathrm{TU}}$ denote the class of convex TU games with the player set $N$.

A permutation of the players in $N$ is a bijection $\sigma:\{1, \ldots, n\} \rightarrow N$, where $\sigma(i)$ denotes which player in $N$ is at position $i$, and $\sigma^{-1}(i)$ denotes the position of player $i$. Let $\Sigma^{N}$ denote the set of all permutations on $N$. For a permutation $\sigma \in \Sigma^{N}, P_{i}^{\sigma}=\left\{j \in N \mid \sigma^{-1}(j)<\sigma^{-1}(i)\right\}$ denotes the coalition of players which precede $i$ with respect to the order $\sigma$. In a permutation $\sigma \in \Sigma^{N}, m_{i}^{\sigma}(v)=v\left(P_{i}^{\sigma} \cup\{i\}\right)-v\left(P_{i}^{\sigma}\right)$ denotes the marginal contribution of player $i$ to the preceding players, and $m^{\sigma}(v)=\left(m_{1}^{\sigma}(v), m_{2}^{\sigma}(v), \ldots, m_{n}^{\sigma}(v)\right)$ is the vector of marginal contributions. Shapley (1971) and Ichiishi (1981) characterize convex TU games as follows.

Theorem 2.3. The TU game $(N, v)$ is convex if and only if $m^{\sigma}(v)$ belongs to the core of $(N, v)$ for all $\sigma \in \Sigma^{N}$.

Theorem 2.3 implies directly the following theorem.

Theorem 2.4. If a TU game $(N, v)$ is convex, then it is exact, $\Gamma_{\mathrm{c}}^{\mathrm{TU}} \subseteq \Gamma_{\mathrm{e}}^{\mathrm{TU}}$.

For a TU game $(N, v)$ and a coalition $C \in \mathcal{N}$ the subgame $\left(C, v^{C}\right)$ is obtained by restricting $v$ to subsets of $C$. Following Biswas, Parthasarathy, Potters, and Voorneveld (1999), we define totally exact TU games.

Definition 2.5. A TU game $(N, v)$ is totally exact if for every $C \in \mathcal{N}$ its subgame $\left(C, v^{C}\right)$ is exact.

Let $\Gamma_{\text {te }}^{\mathrm{TU}}$ denote the class of totally exact TU games with the player set $N$. Biswas, Parthasarathy, Potters, and Voorneveld (1999) show the following theorem.

Theorem 2.6. A TU game is totally exact if and only if it is convex, that is $\Gamma_{\mathrm{te}}^{\mathrm{TU}}=\Gamma_{\mathrm{c}}^{\mathrm{TU}}$.

\subsection{Non-transferable Utility Games}

A cooperative game with non-transferable utility (NTU game, for short) $(N, V)$ is a family of sets $V=\langle V(S)\rangle_{S \in 2^{N}}$ satisfying the following assumptions: 
$V(\emptyset)=\emptyset$,

$V(S)=V_{\mathrm{p}}(S) \times \mathbb{R}^{N \backslash S}$, where $V_{\mathrm{p}}(S) \subseteq \mathbb{R}^{S}$, for all $S \in \mathcal{N}$,

$0 \in V(S)$ for all $S \in \mathcal{N}$,

$V(N)$ is closed,

comprehensiveness: if $x \in V(S), y \in \mathbb{R}^{N}, y^{S} \leq x^{S}$, then $y \in V(S)$,

the sets $V_{\mathrm{p}}^{+}(S)=\left\{x^{S} \in \mathbb{R}_{+}^{S} \mid x \in V_{\mathrm{p}}(S)\right\}$ are bounded for all $S \in \mathcal{N}$.

Let $\Gamma^{\mathrm{NTU}}$ denote the set of NTU games with the player set $N$.

The core of an NTU game $(N, V), C(V)$ consists of those elements $x \in$ $V(N)$ for which it holds that there exist no $S \in \mathcal{N}$ and $y \in V(S)$ such that $x^{S} \ll y^{S}$, which by comprehensiveness is equivalent to $x \notin \operatorname{int} V(S)$ for any $S \in \mathcal{N}$. Therefore,

$$
C(V)=V(N) \backslash \bigcup_{S \in \mathcal{N}} \operatorname{int} V(S)
$$

Predtetchinski and Herings (2004) provide the following balancedness condition for NTU games.

Definition 2.7. Consider a convex-valued correspondence $\Pi: \mathbb{R}^{N} \rightarrow \Delta$ with a closed graph. The NTU game $(N, V)$ is $\Pi$-balanced provided that the following condition is satisfied: If $x \in \mathbb{R}^{N}, \pi \in \Pi(x)$, and $\lambda \in \Delta^{\mathcal{N}}$ are such that

$$
\begin{aligned}
& x \in \bigcap_{S \in \mathcal{N}, \lambda^{S}>0} V(S), \\
& \pi^{N}=\sum_{S \in \mathcal{N}} \lambda^{S} \pi^{S},
\end{aligned}
$$

then $x \in V(N)$.

$\Pi$-balancedness is a necessary and sufficient condition for the core in a non-transferable utility game to be non-empty. Let $\Gamma_{\Pi-\mathrm{b}}^{\mathrm{NTU}}$ denote the class of $\Pi$-balanced NTU games with the player set $N$.

For an NTU game $(N, V)$ and a coalition $S \in \mathcal{N}$ a subgame $\left(S, V^{S}\right)$ is obtained by restricting $V$ to subsets of $S$ and projecting these sets on $\mathbb{R}^{S}$, and setting $V^{S}(S)=\operatorname{cl} V_{\mathrm{p}}(S)$ to have a closed payoff set for the grand coalition in the subgame. Let $\Gamma_{\mathrm{t}-\Pi-\mathrm{b}}^{\mathrm{NTU}}$ denote the class of totally $\Pi$-balanced NTU games with the player set $N$, the class of games with a non-empty core in each subgame.

NTU convex games have been defined in five ways. 
Definition 2.8. (Vilkov, 1977) An NTU game $(N, V)$ is ordinally convex if for all $S, T \in \mathcal{N}$ we have $V(S) \cap V(T) \subseteq V(S \cap T) \cup V(S \cup T)$.

Let $\Gamma_{\mathrm{oc}}^{\mathrm{NTU}}$ denote the class of ordinally convex NTU games with the player set $N$. Ordinally convex NTU games have numerous applications. Peleg (1984) transforms a social choice situation with a convex effectivity function into an NTU game which is ordinally convex. Demange (1987) provides two examples: a model of public goods and a production economy with increasing returns to scale; Masuzawa (2003) adds $N$-person prisoners' dilemma games and oligopoly models to this class.

For $S \in \mathcal{N}$ let $V^{\circ}(S)=\left\{x \in V(S) \mid x_{i}=0\right.$ for all $\left.i \in N \backslash S\right\}$ and let $V^{\circ}(\emptyset)=0^{N}$. Note that $V^{\circ}(S)=V_{\mathrm{p}}(S) \times\left\{0^{N \backslash S}\right\}$, for all $S \in \mathcal{N}$.

Definition 2.9. (Sharkey, 1981) An NTU game $(N, V)$ is cardinally convex if for all $S, T \in \mathcal{N}$ we have $V^{\circ}(S)+V^{\circ}(T) \subseteq V^{\circ}(S \cap T)+V^{\circ}(S \cup T)$. set $N$.

Let $\Gamma_{\mathrm{cc}}^{\mathrm{NTU}}$ denote the class of cardinally convex NTU games with the player

To discuss the three marginalistic interpretations of NTU convexity (coalition merge, individual merge and marginal convexity) introduced by Hendrickx, Borm, and Timmer (2002), we need the following definitions. Note that the definitions are slightly modified as Hendrickx, Borm, and Timmer (2002) use a different set of assumptions defining an NTU game. Instead of Assumptions (4)-(9) they assume that $V(S) \subseteq \mathbb{R}^{S}$ and $V(S)$ is closed for all $S \in \mathcal{N}$; they do not define $V(\emptyset)$; and they assume zero normalization and monotonicity.

An NTU game $(N, V)$ is zero normalized if $V(\{i\})=\left\{x \in \mathbb{R}^{N}: x_{i} \leq 0\right\}$ for all $i \in N$; it is monotone if for all $S, T \in 2^{N}$ with $S \subseteq T$, for all $x \in V(S)$ there exists $y \in V(T)$ such that $y^{S} \geq x^{S}$. The set of weakly Pareto efficient allocations for coalition $S \in \mathcal{N}$ is defined by $\operatorname{WP}(S)=\{x \in$ cl $\left.V(S) \mid \nexists y \in V(S): y^{S} \gg x^{S}\right\}$. Let $\alpha_{i}=\sup \left\{x_{i} \mid x \in V(\{i\})\right\}, i \in N$. The set of individually rational allocations for coalition $S \in \mathcal{N}$ is defined by $\operatorname{IR}(S)=\left\{x \in \operatorname{cl} V(S) \mid \forall i \in S: x_{i} \geq \alpha_{i}\right\}$. An NTU game $(N, V)$ is superadditive if for all coalitions $S, T \in \mathcal{N}$ such that $S \cap T=\emptyset$ we have $V(S) \cap V(T) \subseteq V(S \cup T)$; it is individually superadditive if for all $i \in N$ and for all $S \subseteq N \backslash\{i\}$ we have $V(S) \cap V(\{i\}) \subseteq V(S \cup\{i\})$.

Equation (2) in Definition 2.2 of convexity for TU games states that for any coalition $U$, the marginal contribution of $U$ to a coalition is at least equal to U's contribution to a smaller coalition. The same idea in the NTU setting is formulated as follows. 1

\footnotetext{
${ }^{1}$ Note that superadditivity was required in the original definition of Hendrickx, Borm, and Timmer (2002). In our setting it is implied by coalition merge convexity using Assumption (4), $S=\emptyset$, and comprehensiveness.
} 
Definition 2.10. An NTU game $(N, V)$ is coalition merge convex if it is monotone and for all $U \in \mathcal{N}$ and $S, T \in 2^{N}$ such that $S \subsetneq T \subseteq N \backslash U$ the following statement is true: For all $x \in \operatorname{WP}(S) \cap \operatorname{IR}(S)$, all $y \in V(T)$, and all $z \in V(S \cup U)$ such that $z^{S} \geq x^{S}$, there exists a $v \in V(T \cup U)$ such that $v^{T} \geq y^{T}$ and $v^{U} \geq z^{U}$.

Let $\Gamma_{\mathrm{cmc}}^{\mathrm{NTU}}$ denote the class of coalition merge convex NTU games with the player set $N$.

Equation (3) in Definition 2.2 of convexity for TU games says that for any player $i$, the marginal contribution of $i$ to some coalition is at least equal to $i$ 's contribution to a smaller coalition. The analogous concept in the NTU setting reads as follows. ${ }^{2}$

Definition 2.11. An NTU game $(N, V)$ is individual merge convex if it is monotone and for all $j \in N$ and $S, T \in 2^{N}$ such that $S \subsetneq T \subseteq N \backslash\{j\}$ the following statement is true: For all $x \in \operatorname{WP}(S) \cap \operatorname{IR}(S)$, all $y \in V(T)$, and all $z \in V(S \cup\{j\})$ such that $z^{S} \geq x^{S}$, there exists a $v \in V(T \cup\{j\})$ such that $v^{T} \geq y^{T}$ and $v_{j} \geq z_{j}$.

Let $\Gamma_{\mathrm{imc}}^{\mathrm{NTU}}$ denote the class of individual merge convex NTU games with the player set $N$.

We now define the vector of marginal contributions for an NTU game.

Definition 2.12. Consider an NTU game $(N, V)$ and a permutation $\sigma \in \Sigma^{N}$. The vector of marginal contributions $M^{\sigma}(V)$ is defined by

$$
\begin{array}{r}
M_{\sigma(j)}^{\sigma}(V)=\sup \left\{y_{\sigma(j)} \mid y \in V(\{\sigma(1), \ldots, \sigma(j)\}),\right. \\
\left.\forall i \in\{1, \ldots, j-1\}: y_{\sigma(i)} \geq M_{\sigma(i)}^{\sigma}(V)\right\}
\end{array}
$$

for all $j \in\{1, \ldots, n\}$ !

Theorem 2.3 suggests the following convexity notion for NTU games.

Definition 2.13. An NTU game $(N, V)$ is marginal convex if for all $\sigma \in \Sigma^{N}$ we have $M^{\sigma}(V) \in C(V)$.

Let $\Gamma_{\mathrm{mc}}^{\mathrm{NTU}}$ denote the class of marginal convex NTU games with the player set $N$.

The five notions of NTU convexity are not equivalent in general. Hendrickx, Borm, and Timmer (2002) show that ordinal and cardinal convexity

\footnotetext{
${ }^{2}$ Note that individual superadditivity was required in the original definition of Hendrickx, Borm, and Timmer (2002). In our setting it is implied by individual merge convexity using Assumption (4), $S=\emptyset$, and comprehensiveness.

${ }^{3}$ We use the convention $\sup (\emptyset)=-\infty$.
} 
are not related to each other and to the other three types of convexity. They also provide the following theorem on the relation of the last three convexity notions.

Theorem 2.14. If an $N T U$ game $(N, V)$ is coalition merge convex, then it is individual merge convex, that is $\Gamma_{\mathrm{cmc}}^{\mathrm{NTU}} \subseteq \Gamma_{\mathrm{imc}}^{\mathrm{NTU}}$. If an $N T U$ game $(N, V)$ is individual merge convex, then it is marginal convex, that is $\Gamma_{\mathrm{imc}}^{\mathrm{NTU}} \subseteq \Gamma_{\mathrm{mc}}^{\mathrm{NTU}}$.

Proof. Since we use a different set of assumptions defining an NTU game,we provide a new proof.

$\Gamma_{\mathrm{cmc}}^{\mathrm{NTU}} \subseteq \Gamma_{\mathrm{imc}}^{\mathrm{NTU}}$

This inclusion follows directly from Definitions 2.10 and 2.11 .

$\Gamma_{\mathrm{imc}}^{\mathrm{NTU}} \subseteq \Gamma_{\mathrm{mc}}^{\mathrm{NTU}}$

Assume $(N, V)$ is individually merge convex and let $\sigma \in \Sigma^{N}$ be a permutation. We have to show that $M^{\sigma}(V) \in C(V)$. Without loss of generality we can take the permutation to be the identity. The proof proceeds by induction on the cardinality of $N$.

Let $M^{\sigma, k}\left(V^{\{1, \ldots, k\}}\right)$ denote the marginal vector in the subgame $V^{\{1, \ldots, k\}}$ corresponding to the restriction of $\sigma$ to the first $k$ positions.

If $k=1$, then by definition $M^{\sigma, 1}\left(V^{\{1\}}\right) \in C\left(V^{\{1\}}\right)$.

Let $1 \leq k<n$ and assume that $M^{\sigma, k}\left(V^{\{1, \ldots, k\}}\right) \in C\left(V^{\{1, \ldots, k\}}\right) \stackrel{4}{4}$ We want to show that $M^{\sigma, k+1}\left(V^{\{1, \ldots, k+1\}}\right) \in C\left(V^{\{1, \ldots, k+1\}}\right)$. Suppose on the contrary that $M^{\sigma, k+1}\left(V^{\{1, \ldots, k+1\}}\right) \notin C\left(V^{\{1, \ldots, k+1\}}\right)$. Then there is $S \subseteq\{1, \ldots, k+1\}$ and $x \in V^{\{1, \ldots, k+1\}}(S)$ such that $x^{S} \gg\left(M^{\sigma, k+1}\left(V^{\{1, \ldots, k+1\}}\right)\right)^{S}$.

From the definition of $M^{\sigma, k+1}\left(V^{\{1, \ldots, k+1\}}\right)$ it follows that $S \neq\{1, \ldots, k+1\}$ and $S \neq\{k+1\}$. Consider some point $c \in\left\{z \in \mathbb{R}^{\{1, \ldots, k+1\}} \mid z^{S \backslash\{k+1\}} \leq\right.$ $\left(M^{\sigma, k+1}\left(V^{\{1, \ldots, k+1\}}\right)\right)^{S \backslash\{k+1\}}$ and $\left.\forall i \in S \backslash\{k+1\}: z_{i} \geq \alpha_{i}\right\} \cap \partial V^{\{1, \ldots, k+1\}}(S \backslash$ $\{k+1\})$, where again for all $i \in N: \alpha_{i}=\sup \left\{z_{i} \mid z \in V(\{i\})\right\}$. From the induction hypothesis it follows that $k+1 \in S$, moreover $(N, V)$ is individually superadditive, therefore the given $c$ exists.

Point $c$ is on the boundary of $V^{\{1, \ldots, k+1\}}(S \backslash\{k+1\})$, hence $c \in \operatorname{WP}(S \backslash$ $\{k+1\})$. Since $\forall i \in S \backslash\{k+1\}: c_{i} \geq \alpha_{i}, c \in \operatorname{IR}(S \backslash\{k+1\})$. Since $c^{S \backslash\{k+1\}} \leq\left(M^{\sigma, k+1}\left(V^{\{1, \ldots, k+1\}}\right)\right)^{S \backslash\{k+1\}}$, we have that $x^{S \backslash\{k+1\}} \gg c^{S \backslash\{k+1\}}$. Moreover, using individual superadditivity, $\left(M^{\sigma, k}\left(V^{\{1, \ldots, k\}}\right), 0\right) \in V^{\{1, \ldots, k+1\}}$ $(\{1, \ldots, k\})$. Since $V^{\{1, \ldots, k+1\}}$ is individual merge convex, there exists $y \in$ $V^{\{1, \ldots, k+1\}}(\{1, \ldots, k+1\})$ such that $y^{\{1, \ldots, k\}} \geq M^{\sigma, k}\left(V^{\{1, \ldots, k\}}\right)$ and $y_{k+1} \geq$ $x_{k+1}>M_{k+1}^{\sigma, k+1}\left(V^{\{1, \ldots, k+1\}}\right)$, a contradiction to the definition of $M^{\sigma, k+1}$ $\left(V^{\{1, \ldots, k+1\}}\right)$.

\footnotetext{
${ }^{4}$ Notice that a subgame of an individual merge convex game is individual merge convex.
} 
To illustrate the subtle differences between the various notions of NTU convexity, consider the following example of an ordinally convex NTU game which is neither cardinally, nor marginal, thus by Theorem 2.14 nor individual merge, nor coalition merge convex.

Example 2.15. (Hendrickx, Borm, and Timmer, 2002, Example 4.1.) Consider the following NTU game with player set $N=\{1,2,3\}$. Let

$$
\begin{aligned}
V(\{i\}) & =\left\{x \in \mathbb{R}^{3} \mid x_{i} \leq 0\right\} \text { for all } i \in N, \\
V(\{1,2\}) & =\left\{x \in \mathbb{R}^{3} \mid x_{1} \leq 0, x_{2} \leq 2\right\}, \\
V(\{1,3\}) & =\left\{x \in \mathbb{R}^{3} \mid x_{1}+x_{3} \leq 1\right\}, \\
V(\{2,3\}) & =\left\{x \in \mathbb{R}^{3} \mid x_{2}, x_{3} \leq 0\right\}, \\
V(N) & =\left\{x \in \mathbb{R}^{3} \mid \sum_{i \in N} x_{i} \leq 2\right\} .
\end{aligned}
$$

To show that $(N, V)$ is ordinally convex, let $S, T \in \mathcal{N}$ and let $x \in V(S) \cap$ $V(T)$. If $S \subseteq T, T \subseteq S$ or $S \cap T=\emptyset$, then ordinal convexity is easy to check. If $S=\{1,2\}$ and $T=\{1,3\}$, then $x_{1} \leq 0$ and thus $x \in V(S \cap T)$. Otherwise, $\sum_{i \in N} x_{i} \leq 2$, thus $x \in V(S \cup T)$.

Cardinal convexity of $(N, V)$ fails, since $(0,2,0) \in V^{\circ}(\{1,2\})$ and $(0,0,1) \in$ $V^{\circ}(\{1,3\})$, but $(0,2,0)+(0,0,1)=(0,2,1) \notin V^{\circ}(\{1\})+V^{\circ}(N)$.

Marginal convexity of $(N, V)$ is also not satisfied, since the vector of marginal contributions corresponding to $\sigma=(1,2,3), M^{\sigma}(V)=(0,2,0)$ does not belong to the core: coalition $\{1,3\}$ blocks it. Therefore, by Theorem 2.14, $(N, V)$ is neither individual merge, nor coalition merge convex.

We will continue Example 2.15 in Examples 3.3 and 3.5.

\section{Exact NTU Games}

Theorem 2.4 claims that convex TU games are exact. In this section we generalize exactness to the NTU setting and analyze the relationship of NTU exactness and the various notions of NTU convexity.

Definition 3.1. An NTU game $(N, V)$ is $N T U$ exact if for each $S \in \mathcal{N}$ there exists a core allocation $x \in C(V)$ such that $x \in \partial V(S)$.

Let $\Gamma_{\mathrm{e}}^{\mathrm{NTU}}$ denote the class of exact NTU games with the player set $N$. Every TU game $(N, v)$ with $v(S) \geq 0$ for all $S \in \mathcal{N}$ gives rise to an NTU game $(N, V)$ by defining $V(S)=\left\{x \in \mathbb{R}^{N} \mid x(S) \leq v(S)\right\}$ for all $S \in \mathcal{N}$. Note that Assumptions (4)-(9) are satisfied by $(N, V)$. It is a straightforward exercise to verify the following theorem. 
Theorem 3.2. A TU game $(N, v)$ is exact if and only if the corresponding $N T U$ game $(N, V)$ is $N T U$ exact.

Note that if an NTU game $(N, V)$ is NTU exact, then each of its subgames has a core element, since by definition for each $S \in \mathcal{N}$ there exists a core allocation $x \in C(V)$ such that $x \in \partial V(S)$, and $x$ cannot be blocked in the subgame $\left(S, V^{S}\right)$ either. Thus exact NTU games are a subset of totally $\Pi$-balanced games, $\Gamma_{\mathrm{e}}^{\mathrm{NTU}} \subseteq \Gamma_{\mathrm{t}-\Pi-\mathrm{b}}^{\mathrm{NTU}}$.

Next, we check whether the NTU game in Example 2.15 is NTU exact.

Example 3.3. (Example 2.15 continued.) The NTU game $(N, V)$ in Example 2.15 is NTU exact, since $(0,0,2)$ is a core element on the boundary of $V(\{1\}), V(\{2\})$, and $V(\{1,2\}) ;(2,0,0)$ is a core element on the boundary of $V(\{2\}), V(\{3\})$, and $V(\{2,3\})$; and $(1,1,0)$ is a core element on the boundary of $V(\{1,3\})$.

If for all $S \in \mathcal{N}$ all core elements of the subgame $\left(S, V^{S}\right)$ could be extended to the core of the original game by an appropriate choice for the elements outside $S$, then NTU exactness would follow immediately, since core elements of $\left(S, V^{S}\right)$ are on the boundary of $V(S)$. Example 2.15 shows that NTU exactness of an ordinally convex NTU game cannot be demonstrated in this way. The core of the subgame related to coalition $\{1,2\}$ is $\left\{x \in \mathbb{R}^{2} \mid x_{1}=0,0 \leq x_{2} \leq 2\right\}$. Note that only some elements in this core can be extended to the core of the original game: $\left\{x \in \mathbb{R}^{2} \mid x_{1}=0,0 \leq x_{2} \leq 1\right\}$, since if $y_{1}=0,1<y_{2} \leq 2, y_{3}=2-y_{2}$, then coalition $\{1,3\}$ blocks allocation $y$ in the original game.

To analyze the NTU exactness of ordinally convex NTU games we define the notion of a reduced game for the case where one player leaves the grand coalition. This notion of reduced game originates from Greenberg (1985),

Definition 3.4. Take any NTU game $(N, V), n \geq 2$, and a player $i \in N$. Define:

$$
\begin{aligned}
M & =N \backslash\{i\}, m=n-1, \\
\alpha_{i} & =\sup \left\{x_{i} \mid x \in V(\{i\})\right\}, \\
W(S) & =\left\{x \in \mathbb{R}^{M} \mid \exists \beta>\alpha_{i} \text { such that }(x, \beta) \in V(S \cup\{i\})\right\}, S \subseteq M . \\
P(S) & =V_{\mathrm{p}}(S) \times \mathbb{R}^{M \backslash S}, S \subseteq M .
\end{aligned}
$$

Then, the reduced game $(M, U)$ is given by:

$$
U(S)= \begin{cases}\left\{x \in \mathbb{R}^{M} \mid\left(x, \alpha_{i}\right) \in V(N)\right\} & \text { for } S=M \\ \emptyset & \text { for } S=\emptyset \\ W(S) \cup P(S) & \text { otherwise }\end{cases}
$$


The definition of the reduced game is illustrated in the following example. 5

Example 3.5. (Example 2.15 continued.) If player 3 leaves the grand coalition in Example 2.15, then the derived reduced game looks as follows. $U(\{1,2\})=\left\{x \in \mathbb{R}^{2} \mid x_{1}+x_{2} \leq 2\right\}, U(\emptyset)=\emptyset$. Moreover, $W(\{1\})=$ $\left\{x \in \mathbb{R}^{2} \mid x_{1}<1\right\}, W(\{2\})=\emptyset, P(\{1\})=\left\{x \in \mathbb{R}^{2} \mid x_{1} \leq 0\right\}$ and $P(\{2\})=\left\{x \in \mathbb{R}^{2} \mid x_{2} \leq 0\right\}$ imply that $U(\{1\})=\left\{x \in \mathbb{R}^{2} \mid x_{1}<1\right\}$ and $U(\{2\})=\left\{x \in \mathbb{R}^{2} \mid x_{2} \leq 0\right\}$.

Note that the reduced game is not zero normalized and $U(\{1\})$ is open. Moreover, all the core elements of the reduced game $\left\{x \in \mathbb{R}^{2} \mid x_{1}+x_{2}=\right.$ $\left.2,1 \leq x_{1} \leq 2,0 \leq x_{2} \leq 1\right\}$ can be extended to a core element of the original game by setting $x_{3}=\alpha_{3}=0$.

In general, a reduced game is not always an NTU game. However, Greenberg (1985) shows the following lemma about reduced games of ordinally convex NTU games.

Lemma 3.6. Greenberg, 1985) Consider an ordinally convex NTU game $(N, V)$. Then the reduced game $(M, U)$ is an ordinally convex NTU game.

In his proof Greenberg (1985) considers the setting when $V(S) \subseteq \mathbb{R}_{+}^{N}$ instead of $V(S) \subseteq \mathbb{R}^{N}$, for all $S \in \mathcal{N}$, but due to Assumptions (6) and (8) all the arguments can be carried over to our setting.

We show the following theorem.

Theorem 3.7. If an NTU game $(N, V)$ is ordinally convex, then it is NTU exact, $\Gamma_{\mathrm{oc}}^{\mathrm{NTU}} \subseteq \Gamma_{\mathrm{e}}^{\mathrm{NTU}}$.

Proof. The proof proceeds by induction on the cardinality of $N$.

Let $n=1$. If an NTU game $(N, V)$ is ordinally convex, then it is NTU exact, since $\max \{x \mid x \in V(N)\}$ is well defined, is on the boundary of $V(N)$ and belongs to the core.

Assume that the theorem holds for any game with less than $n$ players. We will show that it also holds for $n$ players.

Let $(N, V)$ be an ordinally convex NTU game with $n \geq 2$ players. Consider some coalition $S \subseteq N$. We show that there exists $y \in C(V)$ such that $y \in \partial V(S)$ and thereby prove that $(N, V)$ is NTU exact.

Let $i \in S$ be arbitrarily chosen and let $M=N \backslash\{i\}$. Lemma 3.6 and the induction hypothesis imply that the reduced game $(M, U)$ is NTU exact.

\footnotetext{
${ }^{5}$ One can verify that Lemma 3.6 and Theorem 3.7 remain valid by using $W(S)=\{x \in$ $\mathbb{R}^{M} \mid \exists \beta$ such that $\forall y \in V(\{i\}): \beta>y_{i}$, and $\left.(x, \beta) \in V(S \cup\{i\})\right\}$. This shows that it is really the blocking possibility by the leaving player that matters in the definition of the reduced game.
} 
Then let $x \in C(U)$ be such that $x \in \partial U(S \backslash\{i\})$ if $S \neq\{i\}$, and let $x \in C(U)$ be arbitrary chosen otherwise. Moreover, let $y \in \mathbb{R}^{N}$ be defined by $y^{M}=x$ and $y^{\{i\}}=\alpha_{i}$. Then, in Step I we show that $y \in C(V)$, in Step II we establish that $y \in \partial V(S)$.

Step I, $y \in C(V)$

Since $x \in C(U)$ by definition $x \in U(M)$, that is $y \in V(N)$. First, we show that $y$ cannot be blocked by any coalition $T \subsetneq N$. Suppose to the contrary that there exist $\beta>\alpha_{i}, z \gg x$ and $T \subsetneq N$ such that $(z, \beta) \in V(T)$. We consider two cases: $T=M$ or $T \neq M$.

Case 1: $T=M$. Then $(z, \beta) \in V(M)$ and by comprehensiveness for all $\epsilon>0$ we have that $\left(z, \alpha_{i}-\epsilon\right) \in V(M)$. Also, for all $\epsilon>0$ we have that $\left(z, \alpha_{i}-\epsilon\right) \in V(\{i\})$ by the definition of $\alpha_{i}$. Ordinal convexity implies that $V(M) \cap V(\{i\}) \subseteq V(N)$, thus for all $\epsilon>0$ we have that $\left(z, \alpha_{i}-\epsilon\right) \in V(N)$. Since $V(N)$ is closed, $\left(z, \alpha_{i}\right) \in V(N)$, implying that $z \in U(M)$, contradicting $x \in C(U)$.

Case 2: $T \neq M$. If $i \notin T$, then $z \in P(T)$ and hence $T$ would block $x$ in $(M, U)$, contradicting $x \in C(U)$. If $i \in T$, then $T \backslash\{i\} \neq \emptyset$, since $\beta>\alpha_{i}$ implies $(z, \beta) \notin V(\{i\})$. Therefore, $z \in W(T \backslash\{i\})$, again contradicting $x \in C(U)$.

Next, we show that $y$ cannot be blocked by $N$ either. Otherwise there exist $\beta>\alpha_{i}, z \gg x$ such that $(z, \beta) \in V(N)$. It follows using comprehensiveness that $\left(z, \alpha_{i}\right) \in V(N)$, implying that $\left(z, \alpha_{i}\right) \in U(M)$, again contradicting $x \in C(U)$. Thus $y \in C(V)$.

Note that the construction used shows that all core elements of the reduced game can be extended to core elements of the original game.

Step II. $y \in \partial V(S)$

Recall that $i$ is a member of $S$. If $S=\{i\}$, then $y \in \partial V(\{i\})$ by the definition of $\alpha_{i}$. If $S=N$, then $y \in C(V)$ by Step I, which implies that $y \in \partial V(N)$.

If $S \neq\{i\}$ and $S \neq N$, then $U(S \backslash\{i\})=W(S \backslash\{i\}) \cup P(S \backslash\{i\})$ and $x \in \partial U(S \backslash\{i\})$. So

$$
\begin{aligned}
x & \in \partial(W(S \backslash\{i\}) \cup P(S \backslash\{i\})) \\
& =\operatorname{cl}(W(S \backslash\{i\}) \cup P(S \backslash\{i\})) \cap \operatorname{cl}\left(\mathbb{R}^{M} \backslash(W(S \backslash\{i\}) \cup P(S \backslash\{i\}))\right) \\
& =(\operatorname{cl} W(S \backslash\{i\}) \cup \operatorname{cl} P(S \backslash\{i\})) \cap \operatorname{cl}\left(\mathbb{R}^{M} \backslash(W(S \backslash\{i\}) \cup P(S \backslash\{i\}))\right) \\
& =(\partial W(S \backslash\{i\}) \backslash \operatorname{int} P(S \backslash\{i\})) \cup(\partial P(S \backslash\{i\}) \backslash \operatorname{int} W(S \backslash\{i\})),
\end{aligned}
$$

which implies that there are two (not exclusive) cases:

$x \in \partial W(S \backslash\{i\}) \backslash \operatorname{int} P(S \backslash\{i\})$ or $x \in \partial P(S \backslash\{i\}) \backslash \operatorname{int} W(S \backslash\{i\})$. 
Case 1: $x \in \partial W(S \backslash\{i\}) \backslash$ int $P(S \backslash\{i\})$. Then, $x \in \partial W(S \backslash\{i\})$ implies $x \in \operatorname{cl} W(S \backslash\{i\}) \cap \operatorname{cl}\left(\mathbb{R}^{M} \backslash W(S \backslash\{i\})\right)$. Since $x \in \operatorname{cl} W(S \backslash\{i\})$, there exists a sequence $\left(x^{k}\right)_{k \in \mathbb{N}}$ with $x^{k} \in W(S \backslash\{i\})$ for all $k \in \mathbb{N}$ and $\left(x^{k}\right)_{k \in \mathbb{N}} \rightarrow x$. Then, by the definition of $W(S \backslash\{i\})$, there exists a sequence $\left(\beta^{k}\right)_{k \in \mathbb{N}}$ with $\beta^{k}>\alpha_{i}$ and $\left(x^{k}, \beta^{k}\right) \in V(S)$ for all $k \in \mathbb{N}$. Due to comprehensiveness $\left(x^{k}, \alpha_{i}\right) \in V(S)$ for all $k \in \mathbb{N}$ as well, and the sequence $\left(x^{k}, \alpha_{i}\right)_{k \in \mathbb{N}}$ converges to $\left(x, \alpha_{i}\right)$, implying that $\left(x, \alpha_{i}\right) \in \operatorname{cl} V(S)$. Since $x \in \operatorname{cl}\left(\mathbb{R}^{M} \backslash W(S \backslash\{i\})\right)$ as well, there exists a sequence $\left(x^{k}\right)_{k \in \mathbb{N}}$ with $x^{k} \in \mathbb{R}^{M} \backslash W(S \backslash\{i\})$ for all $k \in \mathbb{N}$ and $\left(x^{k}\right)_{k \in \mathbb{N}} \rightarrow x$, that is for all $\beta>\alpha_{i}$ we have that $\left(x^{k}, \beta\right) \in \mathbb{R}^{N} \backslash V(S)$ for all $k \in \mathbb{N}$. In particular, $\left(x^{k}, \alpha_{i}+1 /(k+1)\right) \in \mathbb{R}^{N} \backslash V(S)$ for all $k \in \mathbb{N}$, and $\left(x^{k}, \alpha_{i}+1 /(k+1)\right)_{k \in \mathbb{N}} \rightarrow\left(x, \alpha_{i}\right)$, implying that $\left(x, \alpha_{i}\right) \in \mathrm{cl}\left(\mathbb{R}^{N} \backslash V(S)\right)$. So $\left(x, \alpha_{i}\right) \in \operatorname{cl} V(S) \cap \operatorname{cl}\left(\mathbb{R}^{N} \backslash V(S)\right)$, thus $y \in \partial V(S)$.

Case 2: $x \in \partial P(S \backslash\{i\}) \backslash$ int $W(S \backslash\{i\})$. By ordinal convexity of $(N, V)$ we have $V(S \backslash\{i\}) \cap V(\{i\}) \subseteq V(S)$, which together with $x \in \partial P(S \backslash\{i\})$ implies that there exists a sequence $\left(x^{k}, \alpha_{i}^{k}\right)_{k \in \mathbb{N}}$ with $\left(x^{k}, \alpha_{i}^{k}\right) \in V(S)$ for all $k \in \mathbb{N}$ and $\left(x^{k}, \alpha_{i}^{k}\right)_{k \in \mathbb{N}} \rightarrow\left(x, \alpha_{i}\right)$, so $\left(x, \alpha_{i}\right) \in \operatorname{cl} V(S)$. Since $x \notin \operatorname{int} W(S \backslash\{i\})$, for all $z \gg x$ and for all $\beta>\alpha_{i}$ we have $(z, \beta) \notin V(S)$. Thus there exists a sequence $\left(x^{k}, \alpha_{i}+1 /(k+1)\right)_{k \in \mathbb{N}} \rightarrow\left(x, \alpha_{i}\right)$ such that $\left(x^{k}, \alpha_{i}+1 /(k+1)\right) \in \mathbb{R}^{N} \backslash V(S)$, implying that $\left(x, \alpha_{i}\right) \in \operatorname{cl}\left(\mathbb{R}^{N} \backslash V(S)\right)$. So $\left(x, \alpha_{i}\right) \in \operatorname{cl} V(S) \cap \operatorname{cl}\left(\mathbb{R}^{N} \backslash V(S)\right)$, thus $y \in \partial V(S)$.

Next, we provide an example of a cardinally convex game which is not NTU exact.

Example 3.8. (A cardinally convex game which is not NTU exact). Con- 
sider the following NTU game with player set $N=\{1,2,3,4\}$. Let

$$
\begin{aligned}
V(\{i\})= & \left\{x \in \mathbb{R}^{4} \mid x_{i} \leq 0\right\}, \quad i \in N, \\
V(\{1,2\})= & \left\{x \in \mathbb{R}^{4} \mid x_{1}+x_{2} \leq 2\right\}, \\
V(\{1,3\})= & \left\{x \in \mathbb{R}^{4} \mid x_{1}, x_{3} \leq 0\right\}, \\
V(\{1,4\})= & \left\{x \in \mathbb{R}^{4} \mid x_{1}, x_{4} \leq 0\right\}, \\
V(\{2,3\})= & \left\{x \in \mathbb{R}^{4} \mid x_{2}, x_{3} \leq 0\right\}, \\
V(\{2,4\})= & \left\{x \in \mathbb{R}^{4} \mid x_{2}, x_{4} \leq 0\right\}, \\
V(\{3,4\})= & \left\{x \in \mathbb{R}^{4} \mid x_{3}, x_{4} \leq 0\right\}, \\
V(\{1,2,3\})= & \left\{x \in \mathbb{R}^{4} \mid x_{1}+x_{2}+x_{3} \leq 4\right\}, \\
V(\{1,2,4\})= & \left\{x \in \mathbb{R}^{4} \mid x_{1}+x_{2}+x_{4} \leq 4\right\}, \\
V(\{1,3,4\})= & \left\{x \in \mathbb{R}^{4} \mid x_{1}, x_{3}, x_{4} \leq 0\right\}, \\
V(\{2,3,4\})= & \left\{x \in \mathbb{R}^{4} \mid x_{2}, x_{3}, x_{4} \leq 0\right\}, \\
V(N)= & \left\{x \in \mathbb{R}^{4} \mid x_{1}+x_{2}+x_{3} \leq 4, x_{4} \leq 0\right\} \\
& \cup\left\{x \in \mathbb{R}^{4} \mid x_{1}+x_{2}+x_{4} \leq 4, x_{3} \leq 0\right\} \\
& \cup\left\{x \in \mathbb{R}^{4} \mid x_{1}+x_{2}+x_{3}+x_{4} \leq 6, x_{1} \leq-1\right\} .
\end{aligned}
$$

The game $(N, V)$ above is cardinally convex, since

(i) $V^{\circ}(\{1,2,3\})+V^{\circ}(\{1,2,4\}) \subseteq V^{\circ}(\{1,2\})+V^{\circ}(N)$ using the third set in the definition of $V(N)$. Notice that to do so we make use of the fact that for $x \in V^{\circ}(N), x_{1}$ and $x_{2}$ can be chosen negatively in order to increase the values of $x_{3}$ and $x_{4}$.

(ii) For all other $S, T \in \mathcal{N}$ it is easy to verify that $V^{\circ}(S)+V^{\circ}(T) \subseteq V^{\circ}(S \cup T)$.

However, $(N, V)$ is not NTU exact, since there is no core allocation on the boundary of $V(\{1,2\})$. To see that, assume that there is an allocation $x \in C(V)$ such that $x \in \partial V(\{1,2\})$. Since $x \in \partial V(\{1,2\})$, we have that $x_{1}+x_{2}=2$. To have a core allocation, $x_{3} \geq 2$ should hold to prevent blocking by coalition $\{1,2,3\}$ and $x_{4} \geq 2$ should hold to prevent blocking by coalition $\{1,2,4\})$. Thus $x$ should be in the third set in the definition of $V(N)$, requiring that $x_{1} \leq-1$, which would be blocked by player 1 .

By Theorem 2.14, to verify whether the marginalistic interpretations of NTU convexity imply NTU exactness, it is enough to analyze marginal convexity.

Theorem 3.9. If an $N T U$ game $(N, V)$ is marginal convex, then it is $N T U$ exact, that is $\Gamma_{\mathrm{mc}}^{\mathrm{NTU}} \subseteq \Gamma_{\mathrm{e}}^{\mathrm{NTU}}$.

Proof. Consider a marginal convex NTU game $(N, V)$, and a coalition $S \in \mathcal{N}$. For exactness we have to show that there is a core element on the 
boundary of $V(S)$. Let $\bar{\sigma}$ be a permutation such that $S \in\{\bar{\sigma}(1),\{\bar{\sigma}(1), \bar{\sigma}(2)\}$, $\{\bar{\sigma}(1), \bar{\sigma}(2), \bar{\sigma}(3)\}, \ldots, N\}$. Since $(N, V)$ is marginal convex, we have that $M^{\bar{\sigma}}(V) \in C(V)$. By definition, $M^{\bar{\sigma}}(V)$ is on the boundary of $V(T)$ for all $T \in\{\bar{\sigma}(1),\{\bar{\sigma}(1), \bar{\sigma}(2)\},\{\bar{\sigma}(1), \bar{\sigma}(2), \bar{\sigma}(3)\}, \ldots, N\}$, thus it is a core element on the boundary of $V(S)$ as well.

Using Theorems 2.14 and 3.9 we have the following corollary.

Corollary 3.10. Each of coalition merge convexity, individual merge convexity and marginal convexity implies exactness in the NTU setting, that is $\Gamma_{\mathrm{cmc}}^{\mathrm{NTU}} \subseteq \Gamma_{\mathrm{imc}}^{\mathrm{NTU}} \subseteq \Gamma_{\mathrm{mc}}^{\mathrm{NTU}} \subseteq \Gamma_{\mathrm{e}}^{\mathrm{NTU}}$

\section{Conclusion}

In this paper we have generalized exactness to games with non-transferable utility to get the class of NTU exact games $\left(\Gamma_{\mathrm{e}}^{\mathrm{NTU}}\right)$. In a game which is NTU exact for each coalition there is a core allocation on the boundary of its payoff set, meaning that this coalition does not necessarily benefit from the gains of forming the grand coalition in an allocation which is robust against all coalitional deviations. We have noted that NTU exact games are a subset of totally $\Pi$-balanced NTU games $\left(\Gamma_{\mathrm{t}-\Pi-\mathrm{b}}^{\mathrm{NTU}}\right)$, having a non-empty core in each of their subgames.

We have shown that the classes of ordinally convex $\left(\Gamma_{\mathrm{oc}}^{\mathrm{NTU}}\right)$, coalition merge convex $\left(\Gamma_{\mathrm{cmc}}^{\mathrm{NTU}}\right)$, individual merge convex $\left(\Gamma_{\mathrm{imc}}^{\mathrm{NTU}}\right)$, and marginal convex $\left(\Gamma_{\mathrm{mc}}^{\mathrm{NTU}}\right)$ NTU games are a subset of NTU exact games. Moreover, we have given an example of a cardinally convex game $\left(\Gamma_{\mathrm{cc}}^{\mathrm{NTU}}\right)$ which is not NTU exact.

Hendrickx, Borm, and Timmer (2002) show that the aforementioned five classes of NTU convex games do not coincide for more than three players. The only general result (Theorem 2.14) is that coalition merge convexity implies individual merge convexity $\left(\Gamma_{\mathrm{cmc}}^{\mathrm{NTU}} \subseteq \Gamma_{\mathrm{imc}}^{\mathrm{NTU}}\right)$, and individual merge convexity implies marginal convexity $\left(\Gamma_{\mathrm{imc}}^{\mathrm{NTU}} \subseteq \Gamma_{\mathrm{mc}}^{\mathrm{NTU}}\right)$.

Theorem 2.6 claims that the class of convex TU games coincides with the class of totally exact TU games. In the NTU setting we do not have such a theorem. Let $\Gamma_{\text {te }}^{\mathrm{NTU}}$ denote the class of totally exact NTU games with the player set $N$, being NTU exact in all of their subgames. Since an ordinally convex game is exact, and all subgames of an ordinally convex game are ordinally convex, we have that $\Gamma_{\mathrm{oc}}^{\mathrm{NTU}} \subseteq \Gamma_{\text {te }}^{\mathrm{NTU}}$. For marginal convex games a similar argument leads to $\Gamma_{\mathrm{mc}}^{\mathrm{NTU}} \subseteq \Gamma_{\mathrm{te}}^{\mathrm{NTU}}$.

However, using our results it is easy to provide counterexamples where NTU total exactness implies none of the NTU convexity notions. For in- 
stance, the NTU game in Example 2.15 is ordinally convex, and as we argued that game is totally NTU exact. But it is neither cardinal, nor marginal, nor individual merge, nor coalition merge convex. So neither cardinal, nor marginal, nor individual merge, nor coalition merge convexity is implied by total NTU exactness in general. Hendrickx, Borm, and Timmer (2000) provide an example (Example 4.6 there) for an NTU game which is marginal convex but not ordinally convex. That example can be used to show that total NTU exactness does not imply ordinal convexity either.

We summarize the relationships between the various classes of NTU games for more than three players in Figure 1.

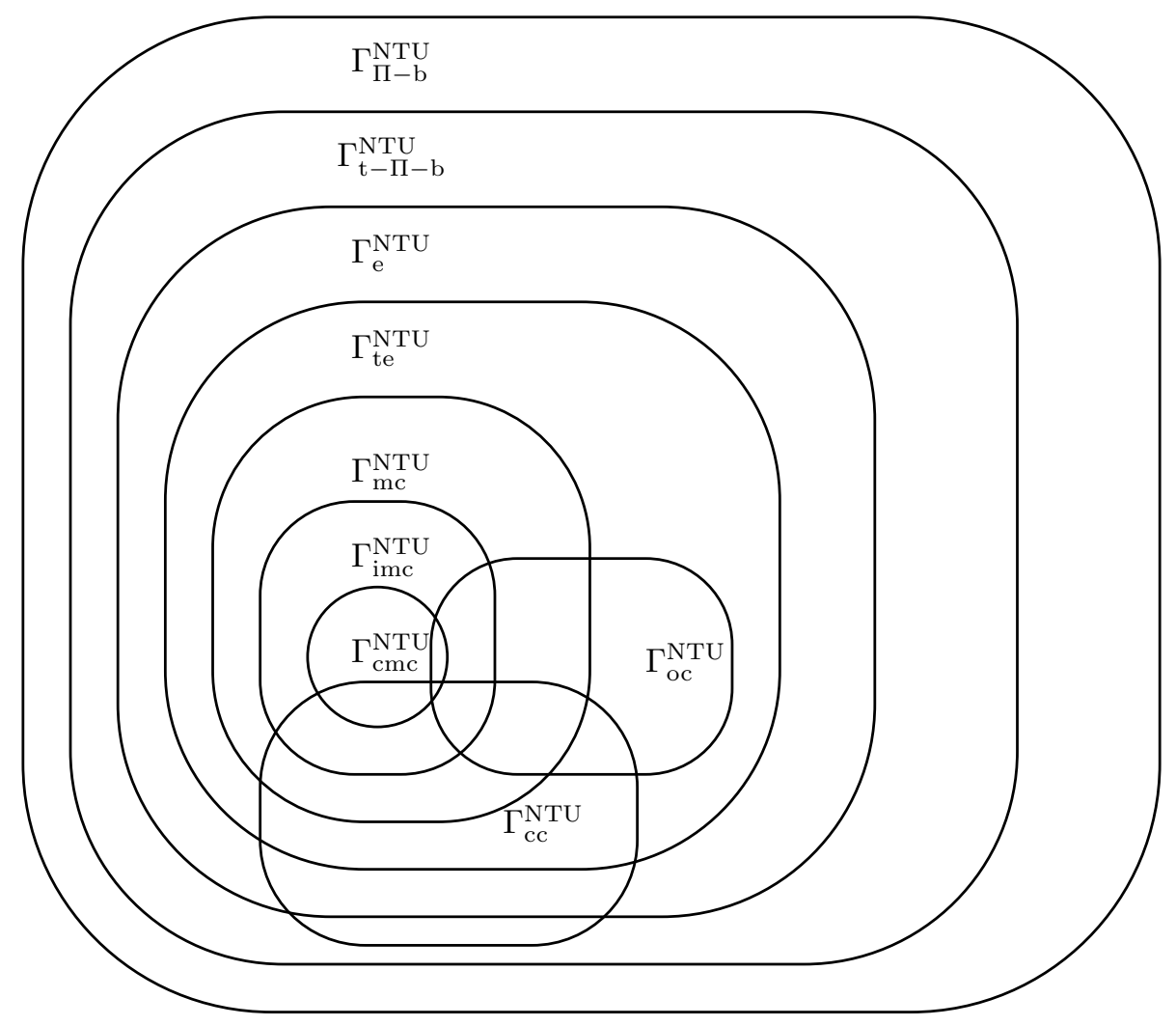

Figure 1: Subsets of П-balanced games.

\section{References}

Aumann, R., and M. Maschler, 1985, Game theoretic analysis of a bankruptcy problem from the Talmud, Journal of Economic Theory 36, 
$195-213$.

Biswas, A. K., T. Parthasarathy, J. A. M. Potters, and M. Voorneveld, 1999, Large cores and exactness, Games and Economic Behavior 28, 1-12.

Calleja, P., P. Borm, and R. Hendrickx, 2005, Multi-issue allocation situations, European Journal of Operational Research 164, 730-747.

Csóka, P., P. J. J. Herings, and L. Á. Kóczy, 2008, Stable allocations of risk, Games and Economic Behavior, doi:10.1016/j.geb.2008.11.001, forthcoming.

Curiel, I., G. Pederzoli, and S. Tijs, 1989, Sequencing games, European Journal of Operational Research 40, 344-351.

Demange, G., 1987, Nonmanipulable cores, Econometrica 55, 1057-1074.

Granot, D., M. Maschler, G. Owen, and W. Zhu, 1996, The kernel/nucleolus of a standard tree game, International Journal of Game Theory 25, 219244.

Greenberg, J., 1985, Cores of convex games without side payments, Mathematics of Operations Research 10, 523-525.

Hendrickx, R., P. Borm, and J. Timmer, 2000, On convexity for NTU-games, Discussion Paper 108, Tilburg University, Center for Economic Research.

, 2002, A note on NTU convexity, International Journal of Game Theory 31, 29-37.

Ichiishi, T., 1981, Super-modularity: Applications to convex games and the greedy algorithm for LP, Journal of Economic Theory 25, 283-286.

Littlechild, S., and G. Owen, 1973, A simple expression for the Shapley value in a special case, Management Science 20, 370-372.

Masuzawa, T., 2003, Punishment strategies make the $\alpha$-coalitional game ordinally convex and balanced, International Journal of Game Theory 32, 479-483.

Peleg, B., 1984, Game Theoretic Analysis of Voting in Comittees (Cambridge University Press).

Predtetchinski, A., and P. J. J. Herings, 2004, A necessary and sufficient condition for the non-emptiness of the core of a non-transferable utility game, Journal of Economic Theory 116, 84-92. 
Schmeidler, D., 1972, Cores of exact games, Journal of Mathematical Analysis and Applications 40, 214-225.

Shapley, L. S., 1971, Cores of convex games, International Journal of Game Theory 1, 11-26.

Sharkey, W., 1981, Convex games without side payments, International Journal of Game Theory 10, 101-106.

Vilkov, V., 1977, Convex games without side payments (in Russian), Vestnik Leningradskiva Universitata 7, 21-24. 